\title{
Towards resolving the evolution of multi-supernova superbubbles
}

\author{
M. S. Oey ${ }^{1}$ \\ ${ }^{1}$ Department of Astronomy, University of Michigan, 830 Dennison Building, Ann Arbor, MI \\ 48109-1042, USA
}

\begin{abstract}
Interstellar superbubbles generated by multiple supernova explosions are common in star-forming galaxies. They are the most obvious manifestation of mechanical feedback, and are largely responsible for transferring both thermal and kinetic energy to the interstellar medium from the massive star population. However, the details of this energy transfer remain surprisingly murky when individual objects are studied. I will summarize what we currently know about candidate dominant processes on these scales.
\end{abstract}

\section{Introduction}

The previous contributions showed a wealth of H I shells and supershells. How wellestablished is it, that these structures originate from mechanical feedback, namely, winds and supernovae ( $\mathrm{SNe}$ ) from OB associations? If we are to understand how massive star feedback affects galaxies on global scales, then it is essential that we understand the origin and evolution of superbubbles, which are the direct manifestation of feedback. We refer not only to mechanical energy, but also to photoionization, and dispersal of nucleosynthesis products from the SNe and massive stars. In this presentation, I use the definition introduced by You-Hua Chu in the 1980's: a "superbubble" is a shell structure originating from multiple stars, rather than a definition related to its size.

The default model for superbubbles is the standard, adiabatic evolution that assumes a hot $\left(10^{6}+\mathrm{K}\right)$, shock-heated interior whose pressure drives the outer shell growth (Pikel'ner 1968; Castor et al. 1975; Dyson 1977). For this model, the shell parameters are determined by only three input parameters: the mechanical "luminosity" $L$, the ambient density $n$, and the age $t$, e.g.,

$$
\begin{gathered}
R \propto(L / n)^{2 / 5} t^{3 / 5}, \\
v=\frac{d R}{d t} \propto(L / n)^{2 / 5} t^{-2 / 5} .
\end{gathered}
$$

We also must know conditions in the ambient environment, for example, the ambient pressure and density distribution. The above is the simplest, analytic representation of this model, but there is also a large body of work in hydrodynamic simulations of these objects over the past two decades (e.g., Mac Low et al. 1989; Bisnovatyi-Kogan et al. 1989; Palouš et al. 1990; Tenorio-Tagle et al. 1991; Tomisaka 1990; Silich et al. 1996; Gazol-Patiño \& Passot 1999; Strickland \& Stevens 2000).

What evidence do we seek, that "star formation," i.e., massive stars, are responsible for the ubiquitous superbubbles that we see in gas-rich galaxies? Since the adiabatic model is based on the shock-heated interior, we expect gas at multiple temperature phases, including hot, X-ray emitting gas; warm, $10^{4} \mathrm{~K}$ photoionized gas; and also the neutral gas that we have seen. Second, we should also find that objects whose input parameters are known should follow the prescribed evolution above. Third, the statistical properties of the superbubble populations should be consistent with the statistical properties of 
the putative parent star-forming regions. Fourth, and perhaps most obvious, we expect a one-to-one correspondence between the superbubbles and the massive star clusters.

\section{Multi-phase ISM}

As mentioned by Elias Brinks in his talk, we often do see the existence of gas at the different temperatures that are predicted by the adiabatic model. A recent example is the X-ray emission in N51 D (Cooper et al. 2004), along with photoionized $\mathrm{H} \alpha$ emission. Superbubbles were already detected in X-rays by Einstein and ROSAT (e.g., Chu \& Mac Low 1990; Wang \& Helfand 1991). It has long been known that there are two categories of X-ray emission from these objects: X-ray bright, and X-ray dim (Chu et al. 1995). The former show X-ray emission in excess of what is expected from the adiabatic model, and the enhancements are likely caused by secondary SN blastwave impacts to the shell walls (Chu \& Mac Low 1990; Oey 1996).

$\mathrm{H}$ recombination emission is generally consistent with photoionization by the observed early-type stars, although density-bounding and shock-heating contributions are also often factors (Hunter et al. 1995; Oey et al. 2000). We usually see $\mathrm{H} \alpha$ emission on the interior of H I shells, consistent with the model that star formation is responsible for the superbubbles. Kim et al. (1999) studied the H I shells of the Large Magellanic Cloud (LMC) and were even able to suggest an evolutionary sequence defined by relative $\mathrm{H} \alpha$ and $\mathrm{H}$ I morphology.

Intermediate-temperature ions are also present. C IV and Si IV, are often seen in absorption in the lines of sight toward massive stars within superbubbles (Chu et al. 1994). Recently, O VI was also detected by FUSE in the line of sight toward one LMC superbubble (Danforth \& Blair 2006).

\section{Dynamics of individual superbubbles}

If the input mechanical power, ambient conditions, and age of the superbubbles can be determined, we can test for consistency with the adiabatic evolution. Such studies are possible for objects in the Milky Way and Magellanic Clouds, where the stellar population can be resolved, and these studies invariably show the existence of a growthrate discrepancy such that the shells are much smaller than predicted by the apparent input parameters (Saken et al. 1992; Brown et al. 1995; Oey \& Massey 1995; Hunter et al. 1995; Oey 1996; Cooper et al. 2004). The problem has been known for many years, and is even seen in single-star nebulae generated by Wolf-Rayet stars (e.g., Cappa et al. 2001, 2005).

What is the current status in resolving this growth-rate discrepancy? There are a number of possible important factors. Those discussed below are the most likely candidates. Combinations of multiple effects may also be at play, including others, such as viscous drag (I. Goldman, private communication), that we do not discuss fully below.

\subsection{Input power overestimated?}

The first possibility for resolving the superbubble growth-rate discrepancy is that the input mechanical luminosity has been overestimated. Stellar wind mass-loss rates $\dot{M}$ are especially suspect, since it has long been suggested that clumping in the winds leads to overestimates in $\dot{M}$ from radio continuum measurements (e.g., Hillier 1991; Nugis et al. 1998). In recent years, this appears to be confirmed by X-ray line profile fitting (Cohen et al. 2006; Miller et al. 2002; Kramer et al. 2003). Fullerton et al. (2006) also find the 
same result from $\mathrm{P}$ v line profiles observed by FUSE. Their analysis of this dominant ion suggests that the overestimates in $\dot{M}$ may be as high as two orders of magnitude in some cases! These overestimates in $\dot{M}$ are relevant primarily to the youngest superbubbles, whose evolution is still dominated by stellar winds instead of SNe, which applies to most dynamical studies of optical or X-ray selected superbubbles.

\subsection{Ambient density underestimated?}

As seen in equations 1.1 and 1.2, an underestimate in the ambient density has an equivalent effect to an overestimate in L. Oey et al. (2002) mapped the immediate environment of three $\mathrm{H} \alpha$-selected LMC superbubbles to determine whether the neutral gas environment was unusually dense. We found an extreme range in conditions for the three objects, with one essentially in an $\mathrm{H}$ I void, another nestled amongst a number of $\mathrm{H}$ I clouds, and the third in a region with no obvious relation to the observed $\mathrm{H}$ I. This lack of any systematic effect suggests that the ambient density may not be the primary source of the growth-rate discrepancy, although it also demonstrates that the ambient environment is more complex than assumed.

We also note that if a superbubble originates in a higher-density cloud, then a miniblowout from the cloud can also accelerate the shell's observed expansion velocity relative to its radius (Oey \& Smedley 1998; Mac Low et al. 1998). Hence it is possible to reproduce unusual observed kinematics in particular objects.

\subsection{Ambient pressure underestimated?}

Another important environment parameter is the interstellar pressure, which counteracts the growth of the shells. In general, models assume a fiducial ambient $P / k$ on the order of $10^{4} \mathrm{~cm}^{-3} \mathrm{~K}$ or less. The various sources of ambient pressure can be broadly described as the thermal pressure, magnetic pressure, turbulent pressure, and cosmic ray pressure. We note that all of these correlate with star-formation rate (SFR). Likewise, superbubbles tend to be prominent in active star-forming galaxies like the LMC and localized active environments. Indeed, the very existence of star-formation in these regions implies higher local pressure (M. Mac Low, private communication). It is therefore plausible that the ambient pressure for superbubbles may have been systematically underestimated.

Oey \& García-Segura (2004) discuss this possibility and present 2-D hydrodynamic simulations of six LMC superbubbles for ambient $P / k=10^{4}$ and $10^{5} \mathrm{~cm}^{-3} \mathrm{~K}$. The models are generated with the same input mechanical luminosity that is estimated from the observed stellar population by Oey (1996). For the lower pressure, the simulated radial density profiles show an extended photoionized morphology that is inconsistent with the observed $\mathrm{H} \alpha$ data. In contrast, the simulations assuming ambient $P / k=10^{5} \mathrm{~cm}^{-3} \mathrm{~K}$ show no extended $\mathrm{H} \alpha$ emission, and an $\mathrm{H} \alpha$ morphology that agrees well with the observations. The observed and modeled velocity structures are similarly more consistent with the models for the high-pressure environment in all cases. The $\mathrm{H}$ I radial profile predictions also differ, and can be used to further test these models.

\subsection{Radiative cooling?}

If the hot superbubble interiors are in fact losing energy by radiative cooling, then the growth will not keep pace with the adiabatic model. Mass-loading into the hot interior via evaporation from the shell walls or ablation of clouds and clumps can drive radiative cooling (e.g., Hartquist et al. 1986; Arthur \& Henney 1996). Alternatively, an increase in metallicity due to the injection of nucleosynthesis products from the parent $\mathrm{SNe}$ can also enhance the cooling rate. Silich \& Oey (2002) show that the X-ray luminosity of a low-metallicity $\left(Z=0.05 Z_{\odot}\right)$ superbubble can be increased by almost an order of 
magnitude, simply by products from $3-4$ SNe. Silich et al. (2001) examine this issue for starburst superwinds. However, the observed X-ray luminosities generally do not appear to suggest anomalous cooling from the superbubble interiors (e.g., Chu et al. 1995).

\subsection{Energy transferred to cosmic rays?}

Another factor whose importance has been underemphasized, is the transfer of superbubble energy to cosmic rays. Multi-SN superbubbles are especially efficient at accelerating cosmic rays because the blastwaves expand into a pre-heated environment (e.g., Parizot et al. 2004). The superbubble interiors thus harbor strong MHD turbulence and magnetic fields (e.g., Bykov 2001; Bykov \& Toptygin 1987), which are needed for cosmic ray acceleration. Because of the multiple SNR shocks, superbubbles also promote multiple accelerations, which can push cosmic rays to higher energies (Parizot et al. 2004; Klepach et al. 2000). The cosmic ray energy distributions and isotope abundances are broadly consistent with superbubble origins. While the role of superbubbles in explaining cosmic ray properties has been recognized for some time, the effect of energy transfer on the parent objects themselves has only been discussed recently, and rough estimates suggest that a few tenths of superbubble kinetic energy could be lost to cosmic rays (Parizot et al. 2004; Bykov 2001). For individual young SNRs, simulations predict growth deviations $\gtrsim 10 \%$ and reduced X-ray luminosities (Ellison et al. 2004). This energy sink applies more to $\mathrm{SN}$-dominated superbubbles, rather than stellar wind-dominated objects.

\section{Properties of global mechanical feedback}

Another approach to evaluating the adiabatic model is to compare observations and predictions for the statistical properties of superbubbles in galaxies, based on the known global star-formation properties. Oey \& Clarke (1997) derived size distributions and expansion velocity distributions for extremes in star-formation history and star-cluster mass functions. The latter, which produces the $\mathrm{H}$ II region luminosity function, is generally a robust power law with a dependence of $L^{-2}$ in differential form (e.g., Efremov \& Elmegreen 1997; Oey \& Clarke 1998). This yields a differential size distribution,

$$
N(R) d R \propto R^{-3} d R
$$

and corresponding distribution in expansion velocities,

$$
N(v) d v \propto v^{-7 / 2} d v .
$$

We find excellent agreement with these relations for the $\mathrm{H}$ I shell population found by Staveley-Smith et al. (1997) for the SMC. More recently, Hatzidimitriou et al. (2005) updated the SMC shell catalog and re-examined these relations, inferring a different star-formation history, but one that is still consistent with a star-formation origin for the structures, plus adiabatic evolution. H I shell size distributions for a few other galaxies also have been examined, for example, the Milky Way (S. Ehlerovà, these proceedings), LMC (Kim et al. 1999), M31, M33, Holmberg II (Oey \& Clarke 1997), and NGC 2403 (Thilker et al. 1998; Mashchenko et al. 1999). These other catalogs are generally consistent with equation 4.1 , but the statistics are more incomplete.

Analysis of the global size distributions leads to the definition of a critical starformation rate, above which the shells merge and shred the neutral ISM, generating pressure-driven outflows from their parent galaxy disks (Clarke \& Oey 2002):

$$
\mathrm{SFR}_{\text {crit }}=0.15 \mathrm{M}_{\mathrm{ISM}, 10} \sigma_{v, 10}^{2} / f_{d} \quad \mathrm{M}_{\odot} \mathrm{yr}^{-1}
$$

where $\mathrm{M}_{\mathrm{ISM}, 10}$ and $\sigma_{v, 10}$ are the mass of the ISM in units of $10^{10} \mathrm{M}_{\odot}$ and the thermal 
velocity dispersion in units of $10 \mathrm{~km} \mathrm{~s}^{-1}$, respectively. The geometric correction factor $f_{d}$ is on the order of unity. Is this expected shredding of the neutral ISM for high SFR morphologically apparent in the $\mathrm{H}$ I datasets?

We can compare the H I maps and shell catalogs for the LMC (Kim et al. 1999) and SMC (Staveley-Smith et al. 1997). The two surveys were both carried out with the Australia Telescope, at similar depth and spatial resolution. Although the LMC is a larger galaxy and has a much higher star-formation rate than the SMC, the number of coherent H I shells identified in the LMC survey is only 126 , roughly one-quarter of the 509 (Hatzidimitriou et al. 2005) found in the SMC. Ordinarily, we would expect a much larger number of shells in the LMC than in the SMC. Morphologically, there is a noticeable contrast between these two galaxies: the LMC H I has a filamentary appearance, consistent with shredding and compression, whereas the SMC has a more quiescent, smoother appearance. It seems surprising that the SMC dataset yields a so much larger shell catalog than the LMC. It turns out that for the LMC, SFR/SFR whereas for the SMC, SFR/SFR crit $\sim 0.1$ (Oey 2001). It will be interesting to examine shell catalogs and properties for larger samples of galaxies, from the THINGS H I survey (E. Brinks, these proceedings), for example.

\section{Detailed correspondence with star-forming regions}

Last, but not least, we seek a one-to-one correspondence between the observed superbubbles and parent star clusters. To date, results are not as clear-cut as is desirable, but there does appear to be broad consistency, though somewhat controversial. Kim et al. (1999) find enough correspondence between the LMC H I and $\mathrm{H} \alpha$ data to suggest an evolutionary sequence based on the morphological and kinematic relationship between these. Hatzidimitriou et al. (2005) examine the spatial relationship between the H I shells in the SMC and existing data for young stellar clusters, and do find broad correspondence, although the quantitative significance of the correlations is ambiguous. They also find that about $10 \%$ of the $\mathrm{H}$ I shells show no counterparts in the stellar population. In Holmberg II, a targeted search for clusters in the H I shells failed to find the expected correspondence at optical wavelengths (Rhode et al. 1999), although $\mathrm{H} \alpha$ and FUV observations seem more consistent with a mechanical feedback origin (Stewart et al. 2000).

Finally, in the theme of this Symposium, triggered star-formation is also an association of massive star clusters with the creation of superbubbles. There are many examples of two-stage, sequential star formation; some well-known examples are N11 (Walborn \& Parker 1992), N44 (Oey \& Massey 1995), and N51 D (Oey \& Smedley 1998; Cooper et al. 2004) in the LMC, and the Rosette Nebula (Williams et al. 1995) in the Galaxy, among others. While it is difficult to establish a causal relationship between two-stage starforming regions, a three-stage sequence is much more convincing. We recently identified the Perseus W3/4 complex in the Galaxy, identified as a triggered system by Thronson et al. (1985), as three-stage, hierarchical star formation, whose morphology is difficult to interpret as anything other than causal, triggered star formation (Oey et al. 2005).

\section{Summary}

To summarize, we see that observations are broadly consistent with mechanical feedback from the most massive stars being responsible for the formation of most superbubbles and shell structures seen in star-forming galaxies, and that these objects can be understood in terms of the standard, adiabatic evolution driven by massive star winds 
and supernovae. There is empirical, multi-wavelength confirmation of the different temperature phases associated with the shock heating of superbubble interiors and also photoionization by $\mathrm{OB}$ stars in the youngest objects, as is qualitatively predicted by the adiabatic model. Statistical properties of shell populations are quantitatively consistent with predicted distributions in size and expansion velocity, based on known global properties of the parent star-forming regions and young star clusters. Above a threshold star-formation rate, we predict interaction among the shells and shredding of the ISM, which appears to be observed in the contrasting $\mathrm{H}$ I morphology and shell populations between the LMC and SMC. The expected one-to-one correlation between young massive-star clusters and superbubbles remains somewhat ambiguous, although a general consistency is tentatively seen.

When comparing the observed evolution and kinematics of individual objects with standard predictions based on detailed knowledge of the parent stellar population and other input parameters, we find that the objects are also broadly consistent with adiabatic evolution, but that the shells are invariably smaller than expected. There are a number of possible reasons for this growth-rate discrepancy. (1) The assumed input power may be overestimated, especially with respect to measured mass-loss rates for stellar winds. (2) It is possible that the ambient density has been systematically underestimated, although our resolved observations of $\mathrm{H}$ I environments for three objects does not especially support this interpretation. (3) There may be a systematic underestimate of the ambient pressure, since the various contributors to interstellar pressure all correlate with star-formation activity. (4) Enhanced radiative cooling may be occurring in the hot superbubble interiors, caused by mass-loading or metallicity enhancements from the parent SNe. (5) Finally, a somewhat-overlooked mechanism is the transfer of mechanical energy from the superbubbles to cosmic rays, which is plausible since superbubbles are an especially effective acceleration environment.

\section{Acknowledgements}

Many thanks to the Symposium organizers and participants, with whom I enjoyed many discussions. This work was supported in part by NSF grant AST-0448893.

\section{References}

Arthur, S. J. \& Henney, W. J. 1996, ApJ 457, 752

Bisnovatyi-Kogan, G. S., Blinnikov, S. I. \& Silich, S. A. 1989, Ap\&SS 154, 229

Brown, A. G. A., Hartmann, D. \& Burton, W. B. 1995, A\&A 300, 903

Bykov, A. M. 2001, Space Sci. Rev. 99, 317

Bykov, A. M. \& Toptygin, I. N. 2001, Astron. Lett. 27, 625

Cappa, C., Niemela, V. S., Martín, M. C. \& McClure-Griffiths, N. M. 2005, A\& A 436, 155

Cappa, C. E., Rubio, M. \& Goss, W. M. 2001, AJ 121, 2664

Castor, J., McCray, R. \& Weaver, R. 1975, ApJ 200, L107

Chu, Y.-H., Chang, H.-W., Su, Y.-L. \& Mac Low, M.-M. 1995, ApJ 450, 157

Chu, Y.-H. \& Mac Low, M-M. 1990, ApJ 365, 510

Chu, Y.-H., Wakker, B., Mac Low, M.-M. \& García-Segura, G. 1994, AJ 108, 1696

Clarke, C. J. \& Oey, M. S. 2002, MNRAS 337, 1299

Cohen, D., Leutenegger, M. A., Grizzard, K. T., Reed, C. L., Kramer, R. H. \& Owocki, S. P. 2006, MNRAS 368, 1905

Cooper, R. L., Guerrero, M. A., Chu, Y.-H., Chen, C.-H. R. \& Dunne, B. C. 2004, ApJ 605, 751

Danforth, C. W. \& Blair, W. P. 2006, ApJ 646, 205

Dyson, J. E. 1977, A\&̊A 59, 161 
Efremov, Y. N. \& Elmegreen, B. G. 1997, ApJ 480, 235

Ellison, D. C., Decourchelle, A. \& Ballet, J. 2004, A\&A 413, 189

Fullerton, A. W., Massa, D. L. \& Prinja, R. K. 2006, ApJ 637, 1025

Gazol-Patiño, A. \& Passot, T. 1999, ApJ 518, 748

Hatzidimitriou, D., Stanimirović, S., Maragoudaki, F., Staveley-Smith, L., Dapergolas, A. \& Bratsolis, E. 2005, MNRAS 360, 1171

Hillier, D. J. 1991, A $\mathscr{E} A$ 247, 455

Hunter, D. A., Boyd, D. M. \& Hawley, W. N. 1995, ApJS 99, 551

Kim, S., Dopita, M. A., Staveley-Smith, L. \& Bessell, M. S. 1999, AJ 118, 2797

Klepach, E. G., Ptuskin, V. S. \& Zirakashvili, V. N. 2000, Astroparticle Phys. 13, 161

Kramer, R. H., Cohen, D. H. \& Owocki, S. P. 2003, ApJ 592, 532

Mac Low, M.-M., Chang, T. H., Chu, Y.-H., Points, S. D., Smith, R. C. \& Wakker, B. P. 1998, ApJ 493, 260

Mac Low, M.-M., McCray, R. \& Norman, M. L. 1989, ApJ 337, 141

Mashchenko, S. Y., Thilker, D. A. \& Braun, R. 1999, A\& A 343, 352

Miller, N. A., Cassinelli, J. P., Waldron, W. L., MacFarlane, J. J. \& Cohen, D. H. 2002, ApJ 577,951

Nugis, T., Crowther, P. A. \& Willis, A. J. 1998, A\&A 333, 956

Oey, M. S. 1996, ApJ 467, 666

Oey, M. S. \& Clarke, C. J. 1997, MNRAS 289, 570

Oey, M. S. \& Clarke, C. J. 1998, AJ 115, 1543

Oey, M. S., Clarke, C. J. \& Massey, P. 2001, in: K. S. de Boer, R.-J. Dettmar \& U. Klein (eds.), Dwarf Galaxies and Their Environment (Shaker Verlag), P. 181

Oey, M. S., Dopita, M. A., Shields, J. C. \& Smith, R. C. 2000, ApJS 128, 511

Oey, M. S. \& García-Segura, G. 2004, ApJ 613, 302

Oey, M. S., Groves, B., Staveley-Smith, L. \& Smith, R. C. 2002, AJ 123, 255

Oey, M. S. \& Massey, P. 1995, ApJ 452, 210

Oey, M. S. \& Smedley, S. A. 1998, AJ 116, 1263

Oey, M. S., Watson, A. M., Kern, K. \& Walth, G. L. 2005, AJ 129, 393

Palouš, J., Franco, J. \& Tenorio-Tagle, G. 1990, A\&\&A 227, 175

Parizot, E., Marcowith, A., van der Swaluw, E., Bykov, A. M. \& Tatischeff, V. 2004, A\&3A 424, 747

Pikel'ner, S. B. 1968, Astrophys. Lett. 2, 97

Rhode, K. L., Salzer, J. J., Westpfahl, D. J. \& Radice, L. A. 1999, AJ 118, 323

Saken, J. M., Shull, J. M., Garmany, C. D., Nichols-Bohlin, J. \& Fesen, R. A. 1992, ApJ 397, 537

Silich, S. A., Franco, J., Palouš, J. \& Tenorio-Tagle, G. 1996, ApJ 468, 722

Silich, S. A. \& Oey, S. 2002, in: D. Geisler, E. K. Grebel \& D. Minniti (eds.), Extragalactic Star Clusters (ASP-CS), p. 459

Silich, S. A., Tenorio-Tagle, G., Terlevich, R., Terlevich, E., \& Netzer, H. 2001, MNRAS 324, 191

Staveley-Smith, L., Sault, R. J., Hatzidimitriou, D., Kesteven, M. J. \& McConnell, D. 1997, MNRAS 289, 225

Stewart, S. G., et al. 2000, ApJ 529, 201

Strickland, D. K. \& Stevens, I. R. 2000, MNRAS 314, 511

Tenorio-Tagle, G., Różyczka, M., Franco, J. \& Bodenheimer, P. 1991, MNRAS 251, 318

Thilker, D. A., Braun, R. \& Walterbos, R. A. M. 1998, A\&̋A 332, 429

Thronson, H. A., Lada, C. J. \& Hewagama, T. 1985, ApJ 297, 662

Tomisaka, K. 1990, ApJ 361, L5

Walborn, N. R. \& Parker, J. W. 1992, ApJ 399, L87

Wang, Q. \& Helfand, D. J. 1991, ApJ 373, 497

Williams, J. P., Blitz, L. \& Stark, A. A. 1995, ApJ 451, 252 


\section{Discussion}

BALLY: Have you considered using momentum conservation (instead of energy conservation) to model shell evolution? It seems that mass-loading and radiative cooling would push the shell towards momentum conservation, implying smaller shell radii.

OEY: It's true that momentum conserving bubbles follow an evolution similar to the adiabatic model. The observations of x-ray emission from superbubbles is some of the strongest evidence favoring the adiabatic evolution, but if the objects in fact undergo radiative cooling, then we should certainly consider momentum conserving models.

Hensler: If you determine the state of superbubbles, then x-ray luminosity, the temperature, metal content, etc., how sensitive is their determination? We found the same growth rate discrepancy e.g., in NGC 1705 (Hensler et al.1997, ApJ)

OEY: Because the x-ray fluxes are relatively low for OB superbubbles, we still do not have adequate quantitative confirmation of the $\mathrm{x}$-ray luminosities and metallicities for such objects. I strongly encourage further observations with XMM and Chandra.

BLITZ: One has to be careful when one attributes causality in star formation from shells. In many most (?) cases, the star formation and molecular clouds that precede them may be independent of the shell that now envelopes the region.

OEY: I completely agree, especially for regions with two-stage sequential star formation. For 3-stage sequential star formation, with the morphology seen in W3/4, I find a conclusion of causality hard to avoid. 